\title{
How Spectral Shapes of Magnetic Energy and Magnetic Helicity Influence their Respective Decay Time Scales
}

\author{
Eric G. Blackman \\ Department of Physics \& Astronomy and Laboratory for Laser Energetics, University of \\ Rochester, Rochester NY 14627; email: blackman@pas.rochester.edu \\ (submitted to, Plasma Phys. and Cont. Fusion)
}

\begin{abstract}
It is shown that in magnetohydrodynamics, the shapes of the magnetic energy and magnetic helicity spectra can influence whether the magnetic helicity resistive dissipation time scale is longer, equal to, or shorter than that of magnetic energy. The calculations highlight that magnetic helicity need not always dissipate significantly more slowly than magnetic energy. While this may be implicitly understood in detailed studies of magnetic helicity in plasma devices, it is not sufficiently emphasized in elementary discussions comparing magnetic helicity vs. magnetic energy evolution. Measuring magnetic energy and helicity decay times may provide a posteriori insight into the initial spectra.
\end{abstract}

PACS codes: 52.30.Cv, 95.30.Qd, 52.65Kj, 52.55 -s, 96.60.Hv, 98.62Mw

\section{Introduction}

Magnetic helicity, (defined by $\int \mathbf{A} \cdot \mathbf{B} d^{3} x$, where $\mathbf{A}$ is the vector potential, $\mathbf{B}$ is the magnetic field and $\int d^{3} x$ is a volume integral) is a measure of the linkage and twist of magnetic field lines, and is a conserved quantity for a closed system in ideal magnetohydrodynamics [1,2]. Magnetic helicity is often more strongly conserved than magnetic energy in the presence of resistive dissipation [2-6]. The concept of a slower decay of magnetic helicity than magnetic energy forms the basis for Taylor relaxation theory [2-6], which describes the end state to which magnetically dominated magnetohydrodynamic configurations relax. The theory is well known in the context of laboratory pinches and has also been applied to the solar corona [7] and astrophysical jets [8]. Taylor theory predicts the relaxed state by minimizing the magnetic energy subject to the constraint that magnetic helicity is conserved. The result is a force-free configuration with the scale of the 
field reaching the largest gradient scale available subject to boundary conditions. Some systems (e.g. Reverse Field Pinches (RFP)) transit toward and away from the Taylor state [e.g. 5], when driven.

The relation between magnetic helicity and magnetic energy decay rates has been studied in [3] but without focusing on the role that energy and magnetic helicity spectra play in their relative magnitudes. Here I specifically show how the relative shapes of the magnetic and magnetic helicity spectra can influence the ratio of magnetic energy to magnetic helicity decay time scales. I derive the spectral shapes of magnetic helicity and magnetic energy for which the magnetic energy decays faster, equal, or more slowly than magnetic helicity. The purpose of these simple calculations is to illustrate very simply how the commonly intuited slow decay of magnetic helicity compared to magnetic energy is influenced by the spectral shapes of these quantities.

\section{Magnetic Helicity vs. Magnetic Energy Decay Time Scales}

In what follows, I will assume that surface terms are small compared to other terms involved in the time evolution of the the magnetic energy, magnetic helicity, and kinetic energies of the system. This would be the case if injection or loss of these quantities through the boundaries proceeds on time scales which are long compared to that of the local dissipation. It should be noted that this is not always the case [5, 9], but the assumption serves to simply illustrate the key concepts for the present purposes, and facilitates straightforward testing with spectral code numerical simulations. The time-evolution equations of magnetic helicity (e.g. [2]), magnetic energy and fluid kinetic energy for incompressible, resistive, viscous MHD can then be written

$$
\begin{gathered}
\partial_{t} H \equiv \partial_{t}\langle\mathbf{A} \cdot \mathbf{B}\rangle=-2 \lambda\langle\mathbf{J} \cdot \mathbf{B}\rangle, \\
\partial_{t} M \equiv \partial_{t}\left\langle\mathbf{B}^{2}\right\rangle=-2 \lambda\left\langle(\nabla \mathbf{B})^{2}\right\rangle-2\langle\mathbf{v} \cdot(\mathbf{J} \times \mathbf{B})\rangle,
\end{gathered}
$$

and

$$
\partial_{t} K \equiv \partial_{t}\left\langle\mathbf{v}^{2}\right\rangle=-2 \nu\left\langle(\nabla \mathbf{v})^{2}\right\rangle+2\langle\mathbf{v} \cdot(\mathbf{J} \times \mathbf{B})\rangle,
$$

where $H$ is the magnetic helicity, $M$ is twice the magnetic energy, $K$ is twice the kinetic energy, $\lambda$ is the magnetic diffusivity, $\nu$ is the viscosity, $\mathbf{v}$ is the velocity, and $\mathbf{B}$ is the magnetic field in Alfvén units. The vector potential $\mathbf{A}$ satisfies $\mathbf{B} \equiv \nabla \times \mathbf{A}$, and the current density is normalized such that $\mathbf{J}=\nabla \times \mathbf{B}$. The brackets indicate a volume average.

In general, the velocity should be included dynamically, as is done in [10]. Here I consider a case in which the velocity term can be approximately ignored in the energy 
dynamics. Deriving a condition for this requires an analysis of the spectra, as discussed in the appendix. Note that even if such a condition were not satisfied, the magnetic energy loss rates and subsequent comparisons to those of magnetic helicity would still be of interest: they would specifically represent the resistive dissipation of magnetic energy, which would not necessarily be approximately equal to the total magnetic energy change rate.

Now write $M=\int_{k_{L}}^{k_{\lambda}} M_{k} d k$ with

$$
M_{k} \equiv \int|\tilde{\mathbf{B}}(\mathbf{k})|^{2} k^{2} d \Omega_{k}=\int k^{2}|\tilde{\mathbf{A}}(\mathbf{k})|^{2} k^{2} d \Omega_{k} \propto k^{-q}
$$

where the tilde indicates the Fourier transform, $\tilde{\mathbf{B}}(\mathbf{k})=i \mathbf{k} \times \tilde{\mathbf{A}}(\mathbf{k}), k$ is the wavenumber magnitude, and $\Omega_{k}$ is the solid angle in wavenumber space. The second relation in (4) follows in the Coulomb gauge $\nabla \cdot \mathbf{A}=0$, and the assumed power-law form of $M_{k}$ takes $q$ to be independent of $k$ between $k_{L}$ and $k_{\lambda}>>k_{L}$, the smallest and largest wave numbers of the system (e.g. the inverse of the system size, and the resistive wavenumber respectively). Similarly, we write the magnetic helicity spectrum

$$
H_{k} \equiv \frac{1}{2} \int\left[\tilde{\mathbf{A}}(\mathbf{k}) \cdot \tilde{\mathbf{B}}^{*}(\mathbf{k})+\tilde{\mathbf{A}}^{*}(\mathbf{k}) \cdot \tilde{\mathbf{B}}(\mathbf{k})\right] k^{2} d \Omega_{k}=M_{k} f(k) / k
$$

where

$$
f(k) \propto k^{-s}
$$

is the assumed form of the fractional magnetic helicity as a function of $k$, with $s$ independent of $k$, and $*$ indicates complex conjugate. In the Coulomb gauge, $f$ is the fractional helicity where $|f| \leq 1$ (realizability condition [11]), and $|f|=1$ is the maximal helicity case. (Ref. [12] provides further discussion in the context of numerical simulations.) We also write the current helicity spectrum $C_{k}=k M_{k} f(k)$. The above definitions imply

$$
\begin{gathered}
\langle\mathbf{A} \cdot \mathbf{B}\rangle=H=\int_{k_{L}}^{k_{\lambda}} H_{k} d k=\int_{k_{L}}^{k_{\lambda}} f(k) M_{k} k^{-1} d k, \\
\langle\mathbf{J} \cdot \mathbf{B}\rangle=C=\int_{k_{L}}^{k_{\lambda}} k^{2} H_{k} d k=\int_{k_{L}}^{k_{\lambda}} f(k) k M_{k} d k, \\
\left\langle\mathbf{B}^{2}\right\rangle=M=\int_{k_{L}}^{k_{\lambda}} M_{k} d k,
\end{gathered}
$$

and

$$
\left\langle(\nabla \mathbf{B})^{2}\right\rangle \simeq \int_{k_{L}}^{k_{\lambda}} k^{2} M_{k} d k
$$

These formulae can be used to calculate the ratio of the time scale of magnetic helicity dissipation to that of magnetic energy dissipation. In general, $f(k)$ could have both positive and negative signs, but for simplicity, we consider an initial condition in which $f(k)$ is 
positive for all $k$ within the integration bounds. Justification comes from 3-D numerical simulations of closed turbulent systems which show that unless kinetic helicity is injected, the magnetic helicity basically maintains the same sign over the spectral range, though transfer between scales does occur $[13,14]$.

For the magnetic helicity dissipation time scale, we then have

$$
\tau_{H}=-H / \partial_{t} H=H / 2 \lambda C=\frac{\int_{k_{L}}^{k_{\lambda}} f(k) M_{k} k^{-1} d k}{2 \lambda \int_{k_{L}}^{k_{\lambda}} f(k) k M_{k} d k},
$$

while for the magnetic energy decay time scale (assuming resistive decay) we have

$$
\tau_{M} \simeq-M / \partial_{t} M \simeq-M /\left(\partial_{t} M\right)_{r e s}=\frac{\int_{k_{L}}^{k_{\lambda}} M_{k} d k}{2 \lambda \int_{k_{L}}^{k_{\lambda}} k^{2} M_{k} d k}
$$

In Fig. 1, the ratio $R \equiv \tau_{H} / \tau_{M}$ is plotted as a function of $q$ and $s$ using (11) and (12), for four different values of $k_{L} / k_{\lambda}$. The plots show a significant parameter regime where $R \leq 1$.

\section{Discussion}

\subsection{Understanding specific regimes analytically}

To better understand Fig. 1, it is useful to further study the ratio $R$ analytically. Using (4) and (6) and assuming $q \neq 1,3$ and $q+s \neq 0,2$, we have

$$
\tau_{H}=\frac{(2-q-s)\left[k^{-(q+s)}\right]_{k_{L}}^{k_{\lambda}}}{2(q+s) \lambda\left[k^{(2-q-s)}\right]_{k_{L}}^{k_{\lambda}}}
$$

and

$$
\tau_{M}=\frac{(3-q)\left[k^{1-q}\right]_{k_{L}}^{k_{\lambda}}}{2 \lambda(1-q)\left[k^{3-q}\right]_{k_{L}}^{k_{\lambda}}}
$$

where the brackets denote taking the difference between the quantity inside evaluated at $k_{\lambda}$ and $k_{L}$. I now explore several different value ranges of $q$ and $s$ and compute $\tau_{M}$ and $\tau_{H}$ and combine the results to determine $R \equiv \tau_{H} / \tau_{M}$.

First consider $\tau_{H}$ : For $0<q+s<2$, Eqn. (16) gives

$$
\tau_{H}=\frac{(2-q-s) k_{L}^{-(q+s)}}{2(q+s) \lambda k_{\lambda}^{(2-q-s)}}
$$


while for $q+s>2$,

$$
\tau_{H}=\frac{(2-q-s)}{2(q+s) \lambda k_{L}^{2}} .
$$

Now consider $\tau_{M}$ : For $1<q<3$, Eqn. (14) gives

$$
\tau_{M}=\frac{(3-q) k_{L}^{1-q}}{2 \lambda(1-q) k_{\lambda}^{3-q}}
$$

while for $q>3$,

$$
\tau_{M}=\frac{(3-q)}{2 \lambda(1-q) k_{L}^{2}}
$$

and for $0<q<1$

$$
\tau_{M}=\frac{(3-q)}{2 \lambda(1-q) k_{\lambda}^{2}}
$$

Combining (15) and (17) for $0<q+s<2$ and $1<q<3$, we have

$$
R=Q\left(k_{\lambda} / k_{L}\right)^{1+s}
$$

where $Q \equiv\left|\frac{(2-q-s)(1-q)}{(q+s)(3-q)}\right|$. Combining (15) and (18) for $0<q+s<2$ and $q>3$, we have

$$
R=Q\left(k_{\lambda} / k_{L}\right)^{q+s-2} .
$$

Combining (15) and (19) for $0<q+s<2$ and $0<q<1$, we have

$$
R=Q\left(k_{\lambda} / k_{L}\right)^{q+s}
$$

Combining (16) and (17) for $q+s>2$ and $1<q<3$, we have

$$
R=Q\left(k_{\lambda} / k_{L}\right)^{3-q}
$$

Combining (16) and (18) for $q+s>2$ and $q>3$, we have

$$
R=Q
$$

Finally, combining (16) and (19), we have for $q+s>2$ and $0<q<1$

$$
R=Q\left(k_{\lambda} / k_{L}\right)^{2}
$$

Three qualitatively different regimes are revealed by (20-25): I. $R<1$ : Eqns. (20) and (21) show cases in which the magnetic helicity can actually decay more quickly than the magnetic energy. Eqn. (20) shows that for $s<-1$ and $0<s+q<2$ and $1<q<3$, $R<1$ can arise. In addition, Eqn. (21) shows that for $0<q+s<2$ and $q>3, R<1$ is 
guaranteed. II. $R \simeq 1$ : Eqn. (24) shows that $R$ is independent of the ratio of wave numbers when $q+s>2$ and $q>3$. For this regime, $Q>1$ so that the magnetic helicity decays more slowly, but $Q$ can be close to 1 , so $R \simeq 1$. III. $R>1$ : For the remaining cases of Eqn. (20) for $s>-1$, and for all regimes of $q, s$ when Eqns. (22), (23) and (25) are applicable, $\tau_{H}>\tau_{M}$ by a factor that depends on $k_{\lambda} / k_{L}$. These three regimes can be seen within the continuous surfaces of Fig. 1, where $R$ was calculated directly from (11) and (12).

In regime III, magnetic energy decays faster than magnetic helicity, and thus will indeed relax toward the Taylor state [3-5]. As just derived, this regime arises when both the magnetic energy and fractional magnetic helicity decrease with increasing $k$, and only when the magnetic energy spectrum is not too steep; regime II rather than regime III applies for a wide range of $s>0$, when $q>3$. Note that in contrast to regimes II and III, regime I can only result from an initial spectrum in which the fractional magnetic helicity increases with decreasing wave number. The realizability condition $(|f(k) \leq 1|)[11]$ then further implies the system is not maximally helical at all scales when case I applies. This in turn implies that there would be scales at which the kinetic energy can equal or dominate the magnetic energy. An example that could qualify for regime I would be one in which the kinetic energy and magnetic energy were equal at large scales, but the velocity falls off more rapidly than the magnetic energy at smaller scales. Whether or not a particular physical phenomenon produces such given spectrum is not addressed here. The main point is just to highlight that the slow resistive decay is of magnetic helicity is not guaranteed in all circumstances.

A subtlety occurs if $f(k)$ were to change rapidly in time (e.g. if its spectral index $s$ changes with time). The estimated $\tau_{H}$ could then be time dependent in such a way that the initial reduction of $H$ by a factor of $1 / e$ could occur in a different parameter regime from the subsequent time evolution of $H$. For example, $H$ could decay to a value of $1 / e$ faster than $M$ reduces by the same fraction so that $R<1$ initially, but the subsequent evolution to $H=0$ might be slower than $M$ if the change in $f(k)$ induces a regime change such that $R>1$ at late times.

In principle, the results above could be used to help diagnose the initial fractional magnetic helicity and magnetic energy spectra for a system in which the decay time scales of these quantities can be measured. The ratio $\tau_{H} / \tau_{M}$ can then be compared with unity and the regime I,II or III revealed. The results can certainly be tested with a 3-D numerical experiment in which magnetic helicity and energy spectra are imposed initially to be in either regime I,II, or III and the time scale for the decay of magnetic energy or magnetic helicity monitored, and a comparison of the resistive decay terms measured. 


\subsection{Relation to previous work}

It is useful to discuss the implications herein in the context of Ref. [3]. The results of the two approaches are compatible, but complementary. Using the Schwartz inequality, Ref. [3] derived an upper bound to the total helicity decay rate as a function of the magnetic energy and magnetic energy decay rate, which was then used to determine how much magnetic helicity $\Delta H$ would decay in the time scale that magnetic energy decays, or, for a given amount of helicity, how long it would take to decay this amount of helicity. No explicit dependence of these decay rates on the spectral indices was considered. In the specific example of a coronal loop considered in Ref. [3], the helicity decayed more slowly than magnetic energy, which was consistent with that example reflecting a more nearly maximal helicity case. Indeed as discussed above, $\tau_{H}>\tau_{M}$ in regime III. But above it was revealed that $\tau_{H}=\tau_{M}$ in regime II and $\tau_{H}<\tau_{M}$ in regime I. These latter two regimes were not explicitly considered in Ref. [3].

The existence of the different regimes suggests that the commonly stated concept that "magnetic helicity decays on a longer time scale than magnetic energy" is not universally applicable. However, the modified statement "magnetic helicity resistively decays on the same or on a longer time scale than magnetic energy" is fully justified when based on a comparison of $H_{\max } / \partial_{t} H$ (or $H_{\max } / \partial_{t} H_{\max }$ ) to $M / \partial_{t} M$, where $H_{\max }$ is the maximum possible magnetic helicity (i.e. corresponding to $f(k)=1$ for all $k$ ) for a given total magnetic energy. In this case, region I does not apply, and only region II and III apply. Note however, that a given system is not guaranteed to have maximal helicity.

A comparison of $H_{\max } / \partial_{t} H$ to $M / \partial_{t} M$ also follows from equation (15) of Ref. [3], the key equation relating the two decay rates using the Schwartz inequality. Using the present notation, the content of that equation is

$$
H_{\max } / \partial_{t} H \geq\left(M / \partial_{t} M\right)^{1 / 2}
$$

which is fully compatible with, but complementary to, the spectral considerations above. It does not by itself directly reveal the role of the spectral shapes.

\section{Conclusions}

Assuming that boundary terms are small, the influence of the spectral shapes of magnetic helicity and magnetic energy on the resistive decay time scales of total magnetic

helicity and magnetic energy was determined. Regimes exist for which the magnetic helicity decays more slowly, more quickly, or at the same rate as magnetic energy. The conventional 
regime for which $\tau_{H} / \tau_{M}>1$ applies to a wide range of spectra, but the existence of regimes for which $\tau_{H} / \tau_{M}=1$ and $\tau_{H} / \tau_{M}<1$, while implicitly understood in some treatments of magnetic helicity evolution in laboratory devices [e.g. 5], are often not emphasized when the basics of magnetic helicity vs. magnetic energy decay are introduced [6]. When the magnetic helicity is nearly maximal, $\tau_{H} / \tau_{M} \geq 1$, with the equality applying only when the magnetic energy spectrum is steep.

Because I have assumed the velocity term in (2) is small (see also the appendix) care must be taken when evaluating the implications for general systems incurring dynamical Taylor relaxation in which velocity fluctuations must be solved for dynamically. Indeed Eq. (2) reveals that the velocity term provides an additional way for magnetic energy to drain into kinetic energy and subsequently dissipate through viscosity. This can help justify the conventional intuition that magnetic helicity decays more slowly than magnetic energy under certain circumstances and it also plays an important dynamical role in driving a system toward and away from the Taylor state [5, 10]. However, since the velocity term can be of either sign, it may also induce a slower decay of magnetic energy depending on the situation. In the presence of dynamically significant velocity fluctuations, the comparison made of time scales in this paper can then be simply thought of as $\tau_{H} /\left(\tau_{M}\right)_{\text {res }}$, where the denominator represents the magnetic energy loss time through resistive dissipation only.

\section{Appendix: On ignoring the velocity term in Eq. (2)}

It is helpful to compare the relative magnitudes of the terms on the right of (2) to motivate ignoring the velocity term. To do so, we write the kinetic energy as

$$
E=\int_{k_{L}}^{k_{\lambda}} E_{k} d k
$$

where $E_{k} \propto k^{-q_{V}}$. We first work on the second term on the right of (2). Noting that $\langle\mathbf{v} \cdot(\mathbf{J} \times \mathbf{B})\rangle \leq\left\langle\left(\mathbf{v}^{2}\right)^{1 / 2} k^{2} \mathbf{B}^{2}\right\rangle$. and using the spectral scalings for kinetic energy combined along with $M_{k} \propto k^{-q}$ from (4), we obtain

$$
\langle\mathbf{v} \cdot(\mathbf{J} \times \mathbf{B})\rangle \lesssim k_{L} B_{L}^{2} v_{L}\left(k_{L} / k\right)^{\frac{2 q+q_{V}-5}{2}}
$$

so that for $q<\frac{5-q_{V}}{2}$

$$
\langle\mathbf{v} \cdot(\mathbf{J} \times \mathbf{B})\rangle \sim k_{L} B_{L}^{2} v_{L}\left(k_{\nu} / k_{L}\right)^{\frac{5-2 q-q_{V}}{2}}
$$

while for $q>\frac{5-q_{V}}{2}$

$$
\langle\mathbf{v} \cdot(\mathbf{J} \times \mathbf{B})\rangle \sim k_{L} B_{L}^{2} v_{L}
$$


In the above equations, the viscous cutoff wavenumber $k_{\nu}$ has entered because I have assumed that the magnetic Prandtl number $\operatorname{Pr}_{M} \equiv \nu / \lambda>>1$ : In magnetically dominated systems such as coronae or laboratory devices, $P r_{M} \simeq\left(T / 1.4 \times 10^{5} \mathrm{~K}\right)\left(n / 10^{16} \mathrm{~cm}^{-3}\right)^{-1}>>1$ [15]. Thus the maximum wavenumber for which $\langle\mathbf{v} \cdot(\mathbf{J} \times \mathbf{B})\rangle$ remains finite is $k_{\nu}>k_{\lambda}$, explaining the appearance of $k_{\nu}$.

For the first term on the right of (2), we again use $M_{k} \propto k^{-q}$ as in (4). We also assume that the decay time at $k_{\lambda}$, the dissipation scale, is approximately equal to the dynamical time at that scale, equivalently,

$$
\lambda k_{\lambda}^{2} \sim k_{\lambda} B_{k_{\lambda}},
$$

remembering that $B$ is in Alfvén units. It follows from some straightforward algebra that

$$
\lambda\left\langle(\nabla \mathbf{B})^{2}\right\rangle \sim k_{L} B_{L}^{3} R_{M}^{\frac{5-3 q}{q+1}},=k_{L} B_{L}^{3}\left(k_{\lambda} / k_{L}\right)^{\frac{5-3 q}{2}},
$$

where the magnetic Reynolds number $R_{M} \equiv B_{L} / k_{L} \lambda$. In this approach, the velocity term in (2) can be dropped when Eqn. (32) >> Eqn. (29) or Eqn. (30), depending on the regime. More specifically, this condition becomes

$$
\frac{B_{L}}{v_{L}}\left(k_{L} / k_{\nu}\right)^{\frac{q-q_{V}}{2}}\left(k_{\lambda} / k_{\nu}\right)^{\frac{5-3 q}{2}}>>1
$$

when $q<\frac{5-q_{V}}{2}$, and

$$
\frac{B_{L}}{v_{L}}\left(k_{\lambda} / k_{\nu}\right)^{\frac{5-3 q}{2}}>>1
$$

when $q>\frac{5-q_{V}}{2}$. Note that the square root of the ratio of magnetic to kinetic energies which enters the above relations is that at the outer scale, not the total, so the total magnetic energy can be larger than the total kinetic energy even the two energies do not satisfy this ordering at any given scale.

To see what the conditions above would imply for a specific example, consider $B_{L} \sim v_{L}$ and let $q_{V}=5 / 3$. Then $\frac{5-q_{V}}{2}=5 / 3$, so for $q<5 / 3$, the condition (33) would always be satisfied in this case. It is common to find steeper magnetic energy spectra when compared to kinetic energy spectra in MHD turbulence $[14,16,17,18]$.

Acknowledgments: Thanks to the referees for their comments which led a number of clarifying revisions. Support from DOE grant DE-FG02-00ER54600 is acknowledged.

1. L. Woltjer, Proc. Nat. Acad. Sci., 44489 (1958).

2. M.A. Berger \& G.B. Field, J. Fluid Mech. 147133 (1984). 
3. M.A. Berger, Geophys. Astrophys. Fluid. Dyn. 3079 (1984).

4. J.B. Taylor, Reviews of Modern Physics, 58, 741 (1986)

5. S. Ortolani \& D.D. Schnack, Magnetohydrodynamics of Plasma Relaxation (World Scientific: Singapore, 1993)

6. P.M. Bellan, Spheromaks, (Imperial College Press, London, 2000)

7. J. Heyvaerts \& E.R. Priest, A.\& Ap., 137, 63, (1984)

8. A. Königl \& A.R. Choudhuri, ApJ, 289, 173, (1985)

9. H Ji.,S. C. Prager, J. S. Sarff, PRL 742945 (1995).

10. E.G. Blackman \& G.B. Field, submitted to Phys. Rev. Lett., (2003), astro$\mathrm{ph} / 0303354$.

11. U. Frisch, A. Pouquet, J. Léorat \& A. Mazure, J. Fluid Mech. 68, 769 (1975).

12. A. Brandenburg, W. Dobler, K. Subramanian, Astron.Nachr. 323, 99 (2002)

13. A. Brandenburg, ApJ, 550, 824 (2001)

14. J. Maron \& E.G. Blackman, ApJL 566, L41 (2002).

15. S. A. Balbus \& J.F. Hawley, Rev. Mod. Phys., 70, 1 (1998).

16. J. Cho, A. Lazarian, \& E.T. Vishniac, 2002, ApJL, 566 L49 (2002).

17. N. Haugen, A. Brandenburg, W. Dobler, ApJL, 597 L141 (2003).

18. J. Maron \& S. Cowley, in press ApJ (2004). 

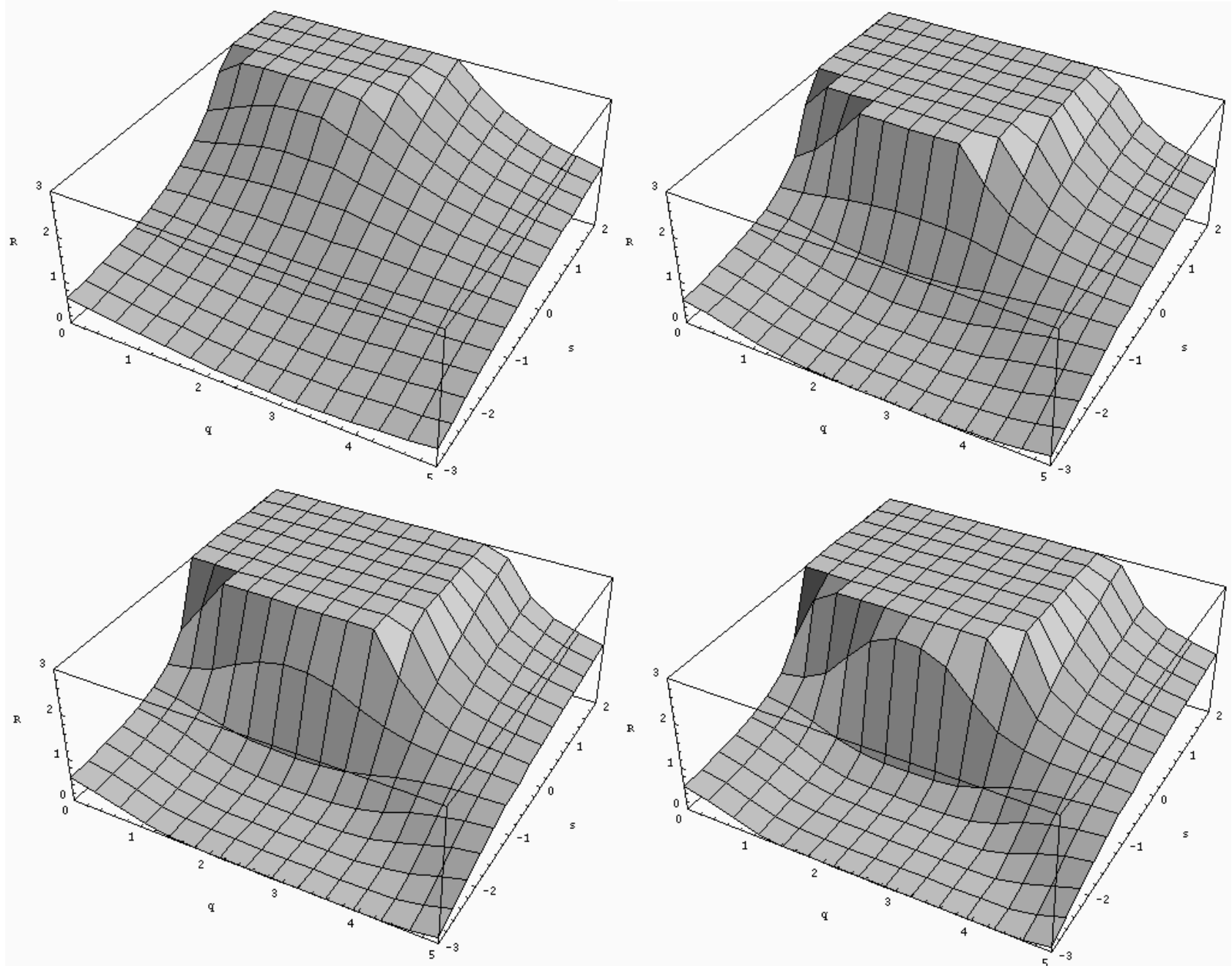

Figure 1: $R \equiv \tau_{H} / \tau_{M}$ vs. fractional helicty spectral index $s$, and energy spectral index $q$. The plots were obtained by taking the ratio of (11) to (12) for 4 different values of $k_{\lambda} / k_{L}$. Clockwise from top left: $k_{\lambda} / k_{L}=10,10^{2}, 10^{4}, 10^{3}$. For $s>0$ and $3>q>0, R>>1$. This is the parameter regime III in the text, and corresponds to the commonly studied regime where magnetic helicity decays more slowly than magnetic energy. However, note that for small $q$ and $s<0, R<1$, and for $q>3$, $R \lesssim 1$. These correspond to regimes I and II discussed below (25). The flat-top surfaces on each of the plots are just an artifact of truncating the vertical axis below the peak values of $R>>1$ attained by the actual solution surfaces. 